\title{
Research on the University Library Information Resource Management Model under the Perspectives of Rich Media and Digital
}

\author{
Chen Zhang \\ Hubei University of Science and Technology Library, \\ Wuhan, Hubei, 430081 China.
}

\begin{abstract}
In this paper, we conduct research on university library information resource management model under the perspectives of the rich media and digital. University library digitization construction can satisfy people thirst for knowledge of information. Although the traditional library is an important place of permanent human cultural heritage, it has many advantages, but its various limitations and unproductive that has far cannot satisfy the people thirst for knowledge of the information. Digital technology brings development opportunities to the traditional library, the information technology revolution promoted the traditional library to computerization, networking with digital development. Traditional library only seize the opportunity, meet the challenges, and strive to change, to modern, the direction of digital that can satisfy the requirement of information society to the library.
\end{abstract}

Keywords- University Library, Information Resource, Management, Rich Media, Digital Feature.

\section{Introduction}

University library user's information needs and services research that is a stereotype often new topic, although the types of library's literature collection in deduction and update, network information is convenient and mass, for the readers in colleges and universities, the demand is eternal, and diverse, dynamic and complex, students whether the user of the library collection quality, work and service quality satisfaction, especially in the current hybrid library environment, obstacles and rights of the reader in the use of the literature information, whether can be solved and satisfied that is the need to research the problem of information demand [1-2].

The general functionality of the digital library could be generally separated into listed two aspects. (1) University library is one of the pillars of its running, with supplementary education and provide the functions of the information retrieval service. With the constant improvement of the degree of the university digital, digital information resources due to its unique convenient, fast advantage of core university teachers and students, become the important resources for teaching and scientific research activities. (2) In the digital environment, university library staff of a large number of documents in the collection, sorting, to make literature has the certain organization, ordering that because of the large information capacity of digital information resources to facilitate the readers multi-channel retrieval consult that become a carrier of the information stored in the library.

Network information resources to a great extent in a state of disorder as information has a great deal of freedom and liberty, lack of the necessary filtering, quality control and management mechanism, network information brings very big trouble for the user to select and use. How fast and convenient to search the information we need on the Internet is facing a big problem. Only the organization and the 
management of the network information resources effectively and will bring real orderly information space, realize information resource utility maximum effect. This is the necessary in library as well as this article focuses on the discussion. The corresponding organization pattern can be generally listed as the follows. (1) Some publications continuity is very strong. Must pay attention to the continuity, as the collection and management and provide service to be very good cohesion, otherwise, attend, lost its continuity that will affect the quality of the entire collection. (2) Attaches great importance to the accuracy of the contents, emphasis on academic value. Library according to the key subject of schools set, determine the scope of a certain subject, academic value, have a certain depth as can reflect the discipline development level and development trends of online academic resources collection objects. (3) Pay attention to information to make the authority of the publisher, credibility. Choosing books, authors, publishing house is a factor that first selects the core issue in choosing journal [3-4].

Huge information resources under the network environment, variety, in addition to the text and the non-text information, such as images, audio, video, software, databases, etc., showing characteristics of multi-types, cross-regional, across languages, but also has characteristics of network transmission and the information resources sharing. Therefore, establishing and perfecting the network information resources, and clarify its legal nature and scope of duties, and figure out the rights of the copyright owner of the network information resources is very necessary.

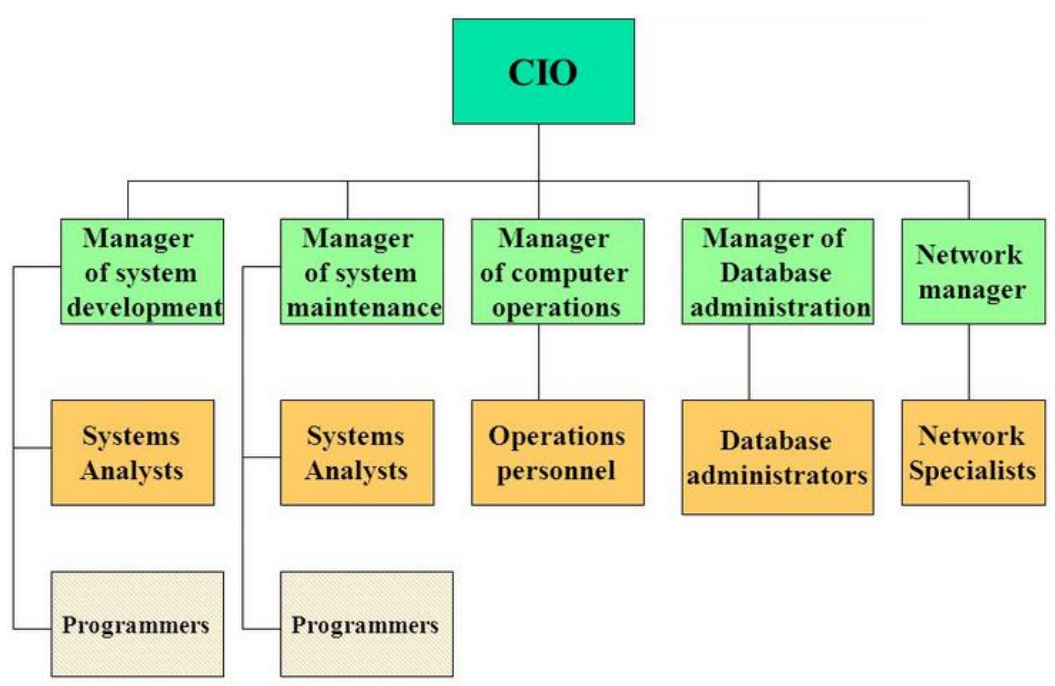

Figure 1. The Architecture of Library Information Resource Management

In this paper, we conduct research on university library information resource management model under the perspectives of the rich media and digital. With the development of the network technology, computer technology and application of the construction of the digital library are increasingly valued, various universities and director of construction of digital library. Construction of digital library in the colleges and universities should first clarify the meaning of digital library and the role of the digital library in colleges and universities, and secondly, the construction of concrete and effective measures is the key to the construction of digital library which will be discussed later.

\section{Our Proposed Methodology}

The Principles of Digital Library. Digital library is the highly value images, text, language, audio, video, film and television, software and 
multimedia information such as the scientific data collection, organization standardization of processing, saving and high quality management, the implementation of knowledge increment and provide high-speed horizontally across the library on the wan connection of electronic access services. Therefore also includes the intellectual property rights, access rights, data security management and so on. Its characteristic is: core collect digital, computerized operation, transmission network, information of liberalization, resources sharing and connecting structure [5].

Characteristics of digital library can be reflected from the listed aspects. (1) It is not only a regional library digitalization construction, several digital libraries, but involves the whole culture resources, mainly is the realization of the whole society of literature information resources sharing, realize to the popularity of the information of the whole society culture, to spread advanced culture for the purpose. (2) In today's traditional media to be being washed out gradually, and build on the express network library digitization construction, keep up with the pace of The Times, to take the network as the basic carrier, spread information culture, that is to say, if we can't realize the core networked information transmission, digital will lose its relying on and original meaning. (3) Literature information resource digitalization is the use of modern science and technology, the traditional literature information to the digital processing, it contains a wide range, including not only the individual literature information digitalization, also includes whole literature information resources digital. (4) Information resource is relative to the energy resources, material resources of the physical form of social wealth, it and energy resources, material resources together constitute the three pillars of the modern social and economic development. Digital transmission can make maximum dissemination and use of the core information resources, and more to save time and money.
Digital library's reader service method is mainly adopted by digital information and technology, it fundamentally changes the information storage, processing, retrieval, transfer and management way, it is the main characteristics of the production, storage and management of digital resources, transport and services, covering multiple distribution mode. Because digital library is a distributed, so the reader can through computer network of crossdatabase search for information and the information we need. It is in accordance with the unified information standard and orderly organization of electronic information, has strong information release and communication functions. Digital library to run at a high speed broadband network as the tool, provide readers with quick, efficient and comprehensive services. Like industrial economy is dependent on the transport and energy, the digital library is also a high-tech economic infrastructure and a necessary condition [6-7].

Knowledge economy is based on knowledge and information of the production, storage, use and consumption, and all kinds of information collected by digital libraries are indispensable for the entire course of knowledge economy and the digital library digital collection with high and new technology, can spread economic, very convenient use by people, thus constantly stimulate people's imagination and creativity that will promote continuous improvement of the whole nation culture quality. Digital library is, as it were, economic and cultural carrier and catalyst in the future, is the direction of future library development as is the realization of knowledge innovation and knowledge economy the main infrastructure and the necessary conditions.

\section{The Library Resources Management.} Library information resources is to point to in the process of human social activities, acquisition, development and the accumulated knowledge as the core of all kinds of information collection, including both physical have paper medium, the 
magnetic medium, light medium such as the carrier of books, periodicals, literature resources and archives resources, but also the type of products of reality have electronic publications and professional database, knowledge base and logic of network database, and can only access of the information public and of the network information resources and people to master the skills and experience, such as intellectual information resources. Knowledge management is a primary extension of the information resources management. Information resources management to the user's information demand as the starting point, through the analysis of information sources, to information acquisition and conversion, information organization, information storage, information retrieval, information development and information transfer, finally to meet the user's information demand, the core of the information resources management is a kind of knowledge is regarded as the theory and methods of information resources management.

Network information organization is based on the characteristics of information itself, the use of the various tools and methods, according to the certain standards, the information processing, sorting, permutation and combination make ordering, systematic and regular, high-level, thus is advantageous to the information storage, transmission, use and so on, in order to meet the information needs of the people processes and the activities which could be summarized as the follows. (1) Theme tree mode mainly through artificial find information, and will receive all information resources, after screening, classify ground according to the uncertainty in advance primary concept of architecture to organize, establish subject category and categories to build a distinct hierarchy system. (2) Asp technology is related to network text information of organic woven together, with node as the basic unit, hyperlinks way between the nodes are linked together, the text information organization for three-dimensional network structure, the user can start from any node, according to the relationship between information in web, browse and query information from different angles of information organization mode. (3) Database is record of information resources according to the fixed format storage organization, by the user query keywords and the equipping can find the information they need to clues, clues connecting again through information to the corresponding network information resources. (4) Free text mode, mainly for the organization of full-text database that is for unstructured text information is a way of organizing and processing and also is a kind of digital library information service. It automatically by computer processing and organization of information to complete picture reflects a literature [8].

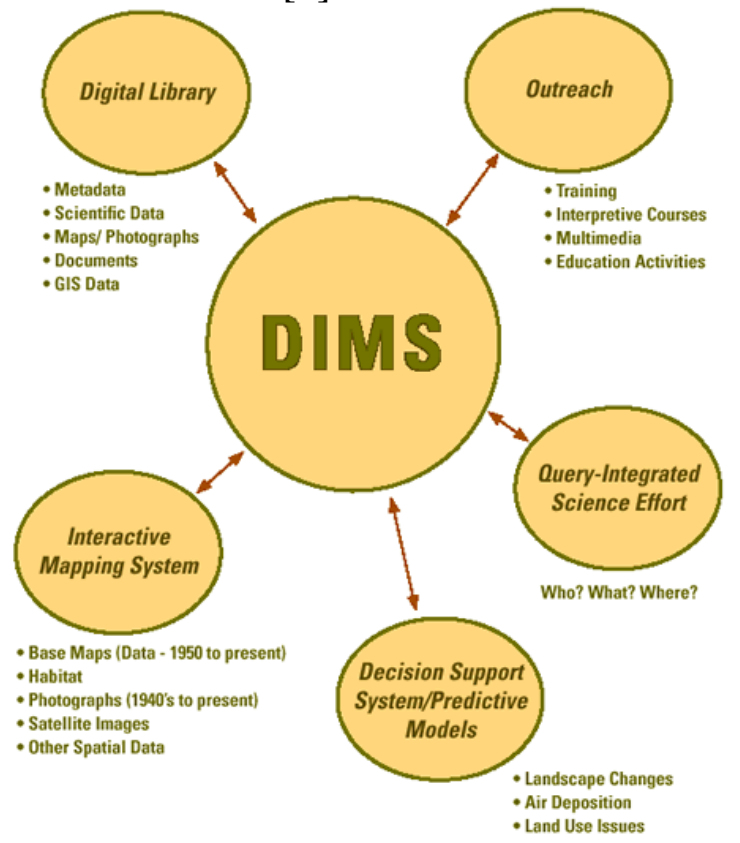

Figure 2. The Digital Library Information Resource Management Principles

The Rich Media and the Digital Combination. Reading promotion is library to deepen service levels and improve service indicators of new request, also is the important of library service function, but also the university library to adapt to the times of the inevitable choice. For now, the work of the library in colleges and universities normal doomed and students read normal reading promotion will 
become the new normal university library service, the new normal truly need time precipitation, and the deepening of the reading promotion practices and accumulation.

The era of the big data, data processing is facing data integration, unity and integration problems of large data. The library data already have the characteristics of big data, the data environment scale, platform structure is complex, large data distributed in the different network nodes after the big data specification faced with how to make all kinds of data and information, and of unity and integration, to build the literature problems with the system of digital resources. Through to the mass, therefore, to research data from heterogeneous resources and all kinds of object data extraction, mapping, harvest, such as import means of collecting, form a unified format, content rich, clear data structure, build all kinds of classification and flexible interface, according to knowledge ontology to organize and reveal, thus ensuring strong efficient retrieval ability and good result relevance ranking.

Under the information environment, with the aid of cloud computing tool and search engines, and so on technology, convenient and timely expresses and show information possible. Reports of library users to social hot spot, the emergence of the new things new ideas as the change of the surrounding environment the details of comprehension and so on aspects of the attention will be more urgent than ever. No matter when and where, on the way to work, dinner, users always hope problems can get answers at any time. In small space words, symbols, pictures, video to the spread of the micro content exactly is a good way to meet the demand of user information timeliness and convenience. Library information service in the future, the border will gradually eliminate the traditional library and digital library, presents a content integration, platform integration, terminal integration, network integration of all media service form. Library should redesign with positive transformation, meet requirements of the national entire media development road.

\section{Conclusion}

In this paper, we conduct research on the university library information resource management model under the perspectives of the rich media and digital. Library as an important indicator of running the colleges and universities, the construction level directly affects the level of running colleges and the universities. In today's computer technology, network communication technology, rapid development of digital technology, promoted the digital construction of the university library. Digital library is the knowledge economy and social development, especially to meet the needs of information highway construction and development of the next generation of new type of network resource organization mode and therefore, accelerating the construction of digital library is the library work in colleges and universities in our country faces a difficult and urgent task. Correspondingly, our research proposes the novel paradigm for the construction which will be meaningful.

\section{ACKNOWLEDGEMENT}

Discipline development forcasting based on WEB OF SCIENCE（No 2015XZ32）

\section{References}

[1] Corrall, Sheila, Mary Anne Kennan, and Waseem Afzal. "Bibliometrics and research data management services: Emerging trends in library support for research." Library trends 61.3 (2013): 636-674.

[2] Kranz, Matthias, Lukas Murmann, and Florian Michahelles. "Research in the large: Challenges for large-scale mobile application research-a case study about NFC adoption using gamification via an app store." International Journal of Mobile 
Human Computer Interaction (IJMHCI) 5.1 (2013): 45-61.

[3] Rahm, Brian G., and Susan J. Riha. "Toward strategic management of shale gas development: Regional, collective impacts on water resources." Environmental Science \& Policy 17 (2012): 12-23.

[4] Sivapalan, Murugesu, Hubert HG Savenije, and Günter Blöschl. "Socio - hydrology: A new science of people and water." Hydrological Processes 26.8 (2012): 1270-1276.

[5] Cowens - Alvarado, Rebecca, et al. "Advancing survivorship care through the National Cancer Survivorship Resource Center." CA: a cancer journal for clinicians 63.3 (2013): 147-150.
[6] Deitz, George D., Susan W. Myers, and Marla R. Stafford. "Understanding consumer response to sponsorship information: A resource - matching approach." Psychology \& Marketing 29.4 (2012): 226-239.

[7] Merz, B., et al. "Charting unknown waters-On the role of surprise in flood risk assessment and management." Water Resources Research $51.8 \quad$ (2015): 6399-6416.

[8] Bertot, John Carlo, Paul T. Jaeger, and Derek Hansen. "The impact of polices on government social media usage: Issues, challenges, and recommendations." Government Information Quarterly 29.1 (2012): 30-40. 